\title{
Consumers' knowledge and attitudes to takeaway food in Merseyside
}

\author{
T.M. Blackham, L. Stevenson, J.C. Abayomi and I.G. Davies \\ Liverpool John Moores University, Faculty of Education, Health \& Community, Barkhill Road, Liverpool, L17 6BD
}

We have shown takeaway food to be energy dense ${ }^{(1)}$, high in $\mathrm{fat}^{(1)}$, saturated $\mathrm{fat}^{(2)}$, salt $^{(3)}$ and sugar ${ }^{(1)}$ and that takeaway food can be reformulated to be healthier without decreasing consumer acceptability ${ }^{(4)}$. Frequent takeaway consumption has also been associated with increased cardio-metabolic risk ${ }^{(5)}$. The mortality rate from cardiovascular disease (CVD) for under $75 \mathrm{~s}$ in Liverpool is greater than the average for England ${ }^{(6)}$, which may be associated with a takeaway food dietary pattern.

There is a dearth of evidence on consumer knowledge and attitudes towards takeaway food. Hence the aim of this study was to investigate takeaway food consumption, knowledge, and attitudes to smaller portion sizes and recipe reformulation in Merseyside. An online questionnaire was distributed to students and staff members at Liverpool John Moores University. All data were initially analysed using descriptive statistics and tested for normality. One-way ANOVA and Pearson correlations were then conducted (statistical significance $p<0 \cdot 05)$. Data from 462 adults were included in the analysis (29\% male, $71 \%$ female; age $18-66)$.

Mean age was $40 \cdot 6$ years (SD 11.2) and mean body mass index (BMI) using self-reported weight and height was $25 \cdot 3 \mathrm{~kg} / \mathrm{m}^{2}(\mathrm{SD}$ 4.8). A quarter of respondents $(25.8 \%$ ) purchased takeaway food less than once a month (infrequent), $48 \cdot 3 \%$ once or twice a month (fairly frequent) and $22 \%$ once or twice a week (frequent). Compared to infrequent takeaway consumers, frequent and fairly frequent takeaway consumers had significantly higher BMI's $(p=0.039 \& p=0.035$ respectively $)$ and were significantly younger $(p<0.001 \&$ $p=0.009$ respectively). Answers were used to generate a total health score based on consumers' takeaway knowledge, such as whether they agreed that takeaway food was low in salt, low in fat or linked with gaining weight. A total receptiveness score was also generated based on questions related to reducing fat, salt, sugar, portion sizes and nutritional labelling. Associations between age, BMI, health score and receptiveness score were correlated (Table).

Table. Pearson correlations. ${ }^{\mathrm{s}}$ significant at the $<0.01$ level.

\begin{tabular}{lcc}
\hline & Total Health Score & Total Receptiveness Score \\
& Correlation coefficient $(r)$ & Correlation coefficient $(r)$ \\
\hline Age & $-0 \cdot 229^{\mathrm{a}}$ & -0.085 \\
BMI & $-0 \cdot 070$ & $0 \cdot 159^{\mathrm{a}}$ \\
Receptiveness & $0 \cdot 177^{\mathrm{a}}$ & \\
\hline
\end{tabular}

Age was significantly associated with health scores; younger takeaway consumers had higher health scores than older consumers. A higher receptiveness score was associated with consumers with higher BMIs. There was also a positive association between health score and receptiveness to change score. Consumers with higher health scores were more receptive to nutritional labelling, smaller portion sizes and purchasing reformulated takeaway foods. Although younger consumers were aware that takeaway food was unhealthy, they were still the most frequent takeaway consumers. This supports existing literature which also found age to be negatively associated with "fast food perceived as unhealthful" and "frequency of fast food intake"(7).

These findings provide evidence that interventions which support takeaway outlets to produce healthier takeaway meals by means of recipe reformulation, combined with the introduction of smaller portion sizes, may be an effective public health measure. Larger studies with a more representative sample of socioeconomic status are warranted. Furthermore, this study supports previous findings by the Food Standards Agency where $58 \%$ of the UK population consumed takeaway meals a few times a month and $22 \%$ at least once a week ${ }^{(8)}$. More evidence on the frequency of takeaway food intake and overall diet composition of takeaway consumers would be useful to determine their associated risk of cardiovascular disease.

1. Jaworowska A, Blackham T, Long R. et al. (2014) Nutrition \& Food Science 44, 414-430

2. Davies I.G, Blackham T, Jaworowska A. et al. (2016) Int J Food Sci Nutr 67, 217-224

3. Jaworowska A. Blackham T, Stevenson L. and Davies I.G. (2012) Appetite 59, 517-522

4. Jaworowska A, Blackham T and Stevenson L. (2011) Proceedings of the Nutrition Society 70, E173

5. Smith KJ, Blizzard L, McNaughton SA. et al. (2012) Eur J Clin Nutr 66, 577-584

6. Public Health England (2015) Health Profile 2015, Liverpool.

7. Dave JM, An LC, Jeffery RW. and Ahlywalia JS. (2009) Obesity (Silver Spring) 17, 1164-70

8. Food Standards Agency (2007) Consumer attitudes to food standards, Wave 7. February 2007. 